\title{
The Neurotoxic Effects of Methotrxate (MTX) on Rat Hippocampus and to Explore the Neuroprotective Role of Alpha Lipoic Acid (ALA): Review Article Muhammad O. Balhaj*, Amal Elshahat Ibrahim, Amira E. Alsemeh, Heba Osama Mohammed Department of Anatomy and Embryology, Faculty of Medicine, Zagazig University, Egypt \\ *Corresponding author: Muhammad O. Balhaj, Mobile: (+20) 01065359115, Email: muhammadbalhaj75@gmail.com
}

\begin{abstract}
Background: The hippocampus is a major component of the mammalian brains and the brains of other vertebrates. It belongs to the limbic system and plays important roles in memory consolidation. Methotrexate (MTX) belongs to the antimetabolite group, which is an antineoplastic group of drugs. MTX has a wide range of therapeutic effects: e.g. at high doses in many malignancies and at low doses in autoimmune diseases such as rheumatoid arthritis and psoriasis, due to its anti-inflammatory and immunosuppressive effects. The therapeutic actions of Alpha Lipoic Acid (ALA) is based on its antioxidant properties including its capacity in regenerating endogenous antioxidants, its metal chelating abilities, its action as a scavenger of reactive oxygen species (ROS) and its capability to repair oxidative damage.

Objective: This work aimed to study the neurotoxic effects of methotrxate (MTX) on rat hippocampus and to explore the neuroprotective role of alpha lipoic acid (ALA).

Methods: The databases were searched for articles published in English in 4 data bases [PubMed - Google scholarScience direct and Egyptian bank of knowledge] and Boolean operators (and, or, not) had been used such as neurotoxic effects of methotrxate and rat hippocampus OR alpha lipoic acid and in reviewed articles.

Conclusion: So, from this review article, it can be concluded that, alpha lipoic acid administration along with methotrxate therapy can ameliorate the harmfull effects of methotrxate on hippocampus.
\end{abstract}

Keywords: Rat hippocampus, Methotrxate, Alpha lipoic acid.

\section{INTRODUCTION}

The hippocampus is a part of limbic system. It is responsible for memory and learning. The blood-brain barrier protects the brain from some drugs but many chemotherapeutic drugs can affect its function by direct or indirect methods ${ }^{(1)}$.

Methotrexate (MTX) is a well-known cytostatic agent used as an essential drug in combination chemotherapy for lymphatic system malignancies. It significantly improves the survival of patients with lymphoma or acute lymphoblastic leukaemia (ALL) and effectively prevents the recurrence of central nervous system (CNS) leukaemia. MTX is an antimetabolite drug that has been found to have beneficial effects on a number of chronic inflammatory diseases. This drug is crucial for prophylaxis and treatment in sanctuary sites, including the CNS (2). Several reports have demonstrated that MTX changes memory ability and interferes with the neurogenesis process in terms of both physiology and pathology, which may cause breakdown of the memory system. Alpha lipoic acid (ALA) has been referred to as the "universal antioxidant" because of its unique antioxidant properties; as a coenzyme present in the mitochondria, ALA can eliminate pathogenic free radicals ${ }^{(3)}$.

ALA acts as an antioxidant in both hydrophilic and lipophilic environments in any cell or tissue type as well as in any subcellular compartments in the body. In addition, it is one of the very few substances that can actually cross the blood-brain barrier, and it does not elicit any serious side effects. Different mechanisms of ALA's positive effects on cognitive function have been suggested, including improvement of memory-related signalling pathways, diminution of oxidative stress, and enhancement of mitochondrial function ${ }^{(4)}$.

This work aimed to study the neurotoxic effects of methotrxate (MTX) on rat hippocampus and to explore the neuroprotective role of alpha lipoic acid (ALA).

\section{I-Hippocampus:}

\section{Anatomy of hippocampus:}

The hippocampus is a main component of human brains and the brains of other vertebrates. It belongs to the limbic system and has important roles in memory. Humans and other mammals have two hippocampi, one in each side of the brain. The hippocampus is situated under the cerebral cortex ${ }^{(5)}$.

In both humans and rats, the hippocampus region of the brain is similar in its anatomical structure and its function in memory formation. The hippocampus of rats is similar to humans as it undergoes age-associated changes and degenerations to its circuits. The volume of the human hippocampus is 100 times that of the rat. Furthermore 10 times that of the monkey ${ }^{(6)}$. 


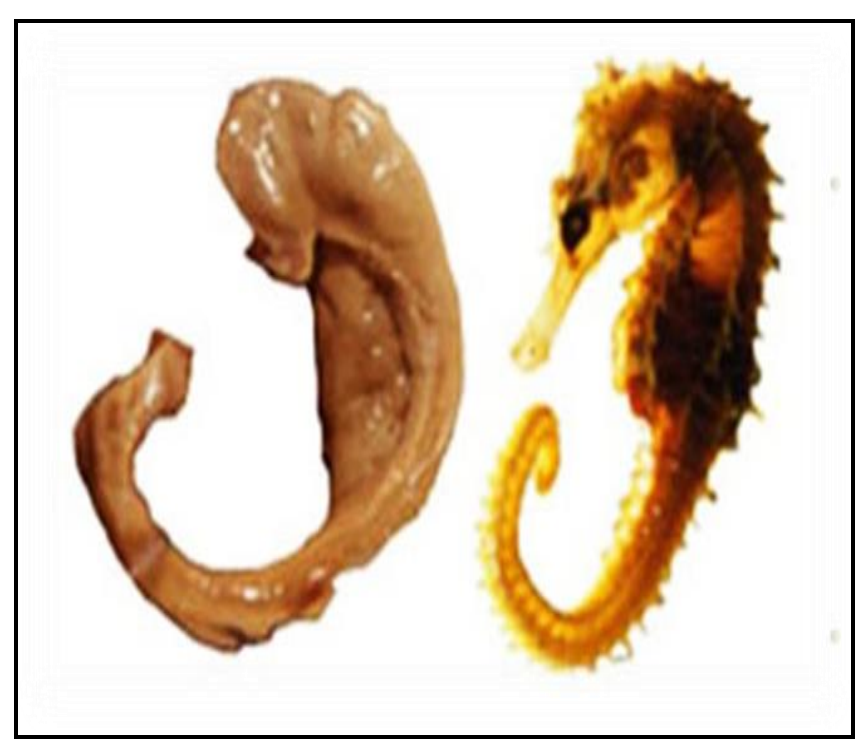

Figure (1): Hippocampus is a neural structure in the medial temporal lobe of the brain that has distinctive curved shape, which resembles a shape of a sea horse (7).

There are three different anatomical terms: hippocampal region, the hippocampal formation and the hippocampus. The hippocampal region is composed of two structures: the hippocampal formation and the para hippocampal region. It's also the central component of the limbic system, which also consists of the olfactory cortex medial to the rhinal fissures, the amygdala, the septum, the mamillary bodies and the anterior thalamic nuclei ${ }^{(8)}$.

The hippocampal formation is described as the complex of six structures; hippocampus proproius, dentate gyrus (DG), pressubiculum, parasubiculum, subiculum proprium and area entorinal cortex (EC). Hippocampus is formed of two parts: cornu ammonis (hippocampus proprius) and dentate gyrus, these two parts are separated by hippocampal sulcus and curve into each other. Despite the dentate gyrus is part of the hippocampus, we can differentiate it from the hippocampus proper by their different cell architecture $^{\left({ }^{9}\right)}$.

In sagittal section, the hippocampus is a Cshaped structure. The name cornu ammonis submits to the Egyptian deity Ammon. The horned shaped of the hippocampus is caused by cell density differentials and the presence of varying degrees of neuronal fibers. The hippocampus presents above the subiculum and medial para hippocampal gyrus, forming a curved elevation, about $5 \mathrm{~cm}$ long, beside the floor of the inferior horn of the lateral ventricle. Its frontal end is enlarged and here its margin may present two or three shallow grooves that provide a paw-like shaped, the pes hippocampi. The DG is a scalloped cortex band, which is related inferiorly to the subiculum, laterally to the hippocampus and medially to the fornix fimbria. Hippocampal fissure presents between DG and subicular extension of para hippocampal. Hippocampus is divided into head, body and tail; head being expanded part while tail is a thin curved part. Cornu ammonis is divided into CA1, CA2, CA3, CA4 and subiculum, which connects hippocampus with entorinal $\begin{array}{llll}\text { cortex } & \text { (Figure } & \text { (10) }\end{array}$

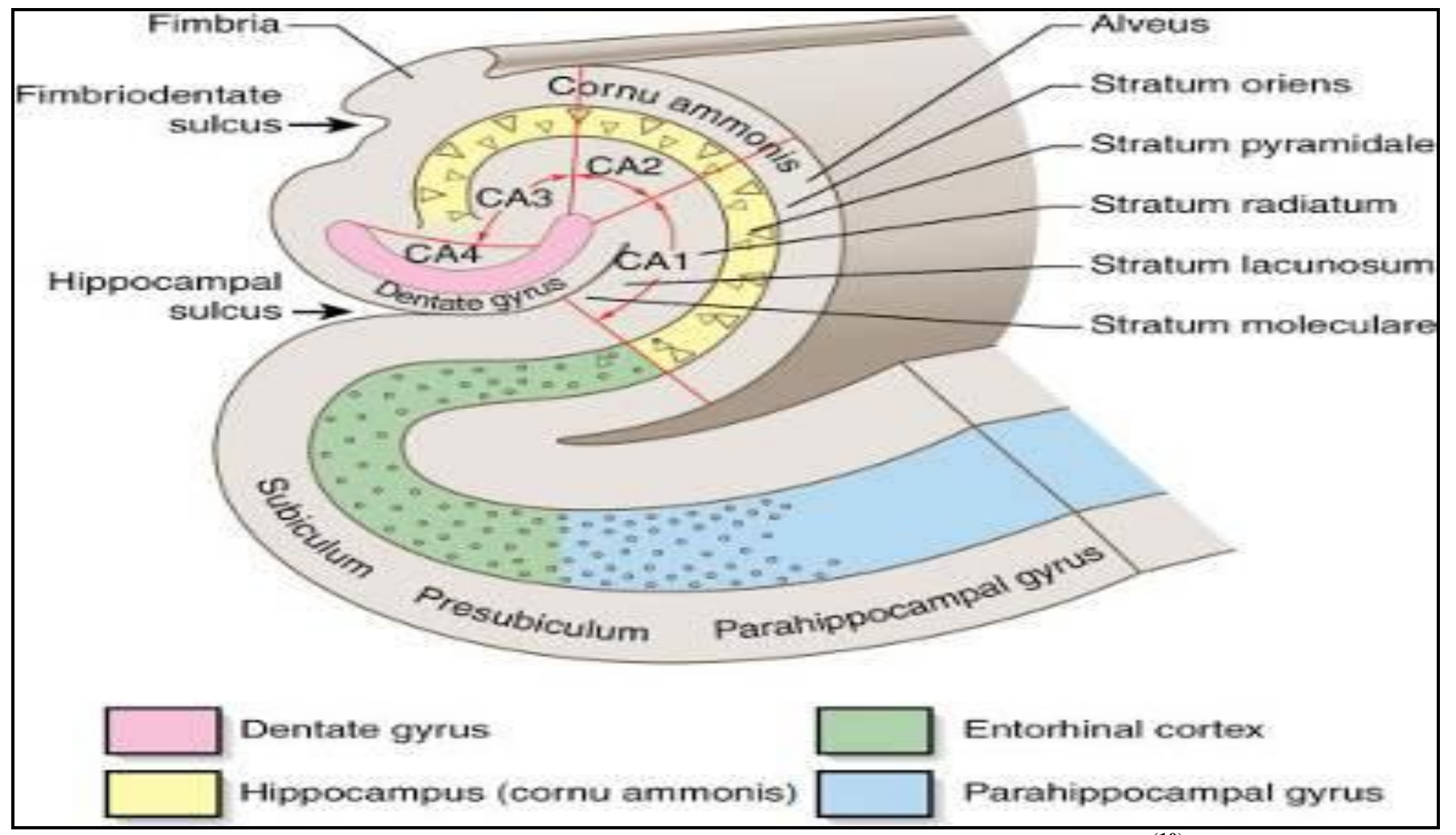

Figure (2): The hippocampal formation in human showing the deposition of varies cell fields ${ }^{(\mathbf{1 0})}$. 


\section{Histology of hippocampus:}

The hippocampus cyto-architecture is organized in regular layers, where the cell bodies are firmly packed together in one layer and the dendrites and the axons reside in other discrete layers with glial cells and interneurons scattered all through. The hippocampus is composed of several subfields. The most regularly terms diverge among the authers are dentate gyrus and the cornu ammonis. The dentate gyrus contains the fascia dentata and the hilus, while cornu ammonis is differentiated into fields CA1, $\mathrm{CA} 2, \mathrm{CA} 3$, and CA4. However, the region known as CA4 is in fact the deep polymorphic layer of the dentate gyrus ${ }^{(\mathbf{1 1})}$.

Hippocampal formation in rat comprises six distinct regions, including the dentate gyrus; hippocampus proper or cornu ammonis (CA) subicular complex (subiculum, pre subiculum, para subiculum) and entorhinalcortex ${ }^{(\mathbf{1 2})}$.

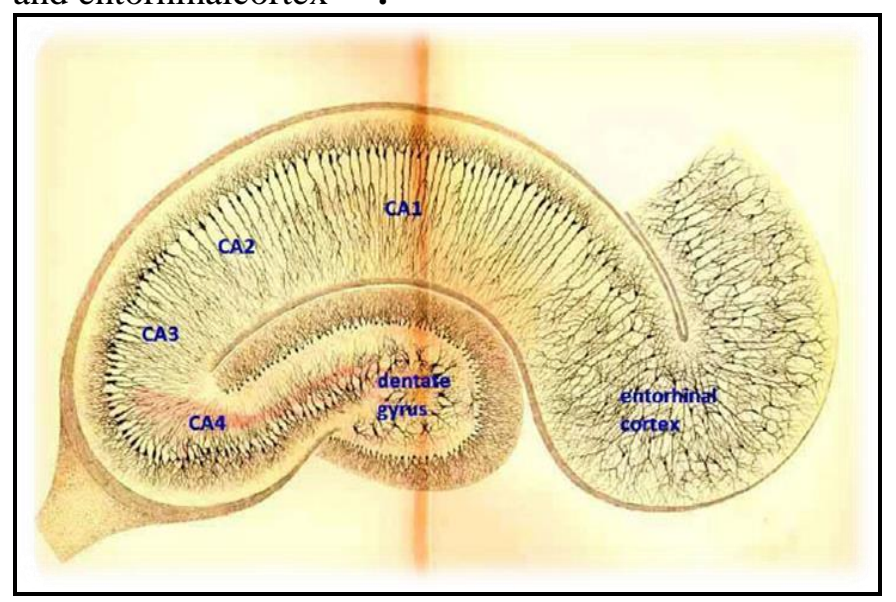

Figure (3): Hippocampus of rat consists of two parts: Cornu ammonis; CA1, CA2, CA3, CA4 (hippocampus proprius) and dentate gyrus ${ }^{(7)}$.

\section{Cytoarchitectural Organization of hippocampus:}

The CA regions are constructed of three clearly defined layers: Polymorphic layer: contains axons of pyramidal neurons, cell bodies of inhibitory basket cells, horizontal trilaminar cells and basal dendrites of pyramidal cells. Pyramidal layer: contains the cell bodies of the pyramidal neurons, which are the principal excitatory neurons of the hippocampus. In CA3 region, this layer contains synapses from the mossy fibers (projections from the dentate gyrus granule cells to CA3). Molecular layer: contains septal and commissral fibers, Schaffer collateral fibers (the projection from CA3 to CA1), some interneurons and perforant path fibers (fibers from entorhinal cortex superficial layers) ${ }^{(\mathbf{1 2})}$.

The laminar organization is similar in all the fields of the hippocampus. The chief cellular layer is called the pyramidal cell layer. This layer is closely packed in CA1 and loosely packed in CA2 and CA3. CA2 contains large and small pyramidal cells. The small pyramidal cells are the same in size to those seen in CA1, while the larger ones are similar in size to those of CA3. Except for its lack of stratum lucidum. The laminar organization of CA2 is considered as a narrow transitional field between $\mathrm{CA} 3$ and CA1. The border between CA 2 and CA3 is characterized by a slight but abrupt widening of the pyramidal cell layer. The border distinguishing CA3 from CA2 isn't well marked ${ }^{(\mathbf{8})}$.

CA4 is the continuation of the CA3 in the concavity of the dentate gyrus. Hilus fasciae dentatae is the term used for the complex of the pyramidal layer of CA4 and the stratum multiform of the gyrus dentatus ${ }^{(12)}$.

The pyramidal cell layer have pyramidal shaped cell bodies and nuclei with single prominent nucleoli, intermingled with glial cells. Cytoplasm of neurons shows rER (rough endoplasmic reticulum) which is seen as Nissl substance. Nissl granules appear in large-sized neurons more than in smallsized neurons. The rER is basically restricted to the cell body but may spread a little into the axonal hillock. Pyramidal cells have a basal dendritic tree, which extends into the stratum oriens and an apical dendritic tree that expands to the hippocampal fissure. There is a narrow, relatively cell-free layer deep to the pyramidal cell layer called the stratum oriens. This layer has the basal dendrites of the pyramidal cells and multiple types of interneurons. On the proximal part of these dendrites the commissural afferents from the contralateral hippocampus are terminated ${ }^{(\mathbf{1 2})}$.

Deep to stratum oriens is the alveus, which contains the myelinated axons of hippocampal pyramidal neurons running in parallel to the stratum pyramidale, the fibers of the alveolar tract from the entorhinal cortex, and projections from the septum. A slight cellular zone termed the stratum lucidum is located in CA3 field, but not in CA2 or CA1. It is presented just above the pyramidal cell layer and is occupied by the mossy fibers of dentate gyrus ${ }^{(8)}$.

Superficial to the stratum lucidum in CA3 and just above the pyramidal cell layer in CA2 and CA1, the stratum radiatum is located. This layer is containing unbranched primary shafts of pyramidal neurons both in the area superior and inferior of Ammon's horn. Schaffer collaterals of axons of pyramidal neurons from CA3 terminate on proximal parts of apical dendrite shafts in CA1. The distal portions of apical dendrite shafts in the stratum radiatum are the field of termination of commissural projections from $\mathrm{CA} 3$ of the contralateral hippocampus and afferents from the septum. This layer is also called the supra pyramidal region. The stratum lacunosum composing of branched apical dendrites of pyramidal neurons. This layer is the terminal field of the perforant path from the entorhinal cortex.The stratum moleculare formed by terminal branches of apical dendrites from the stratum pyramidale ${ }^{(\mathbf{1 3})}$.

Physiology of the hippocampus: 
Hippocampus is a main region of the cerebral cortex engaged in learning, memory and spatial navigation. For learning and memory circle, there are two important pathways: polysynaptic and direct pathway. In the polysynaptic pathway, hippocampus entertains afferent connections from parietal, temporal, and occipital regions through entorhinal cortex and then to dentate gyrus to CA3, CA1, subiculum, alveus, fimbria, fornix, mammillo thalamic tract, anterior thalamus, posterior cingulated and retrosplenial cortex. In the direct intra-hippocampal pathway, hippocampus acquires its input from temporal association cortex via perirhinal and entorhinal regions to CA1. From there projections move via subiculum and entorhinal cortex to inferior temporal cortex, temporal pole, and prefrontal cortex. It is essential to memorize that direct intra-hippocampal pathway is essential in episodic and spatial memory while polysynaptic pathway is essential in the memory ${ }^{(14)}$.

Hippocampus is serious for binding relations among different objects and that relational memory representations can be programed, retrieved and used in service of ongoing cognition. It is a main area provider to language use and processing ${ }^{(14)}$.

The best understanding of the function of the hippocampal formation is there tention of information in short-term memory and its transfer into long-term memory that's mean consolidation of memories. Severe damage to the hippocampus in both hemispheres results in profound difficulties in forming new memories (anterograde amnesia), and often also affects memories formed before the damage (retrograde amnesia). Although the retrograde effect normally extends some years before the brain damage, in some cases older memories remain. This sparing of older memories leads to the idea that consolidation over time involves the transfer of memories out of the hippocampus to other parts of the brain ${ }^{(15)}$.

\section{II-Methotrexate:}

MTX is an anti-metabolite anti cancer drug that has been found to have beneficial effects in a number of chronic inflammatory diseases. Structurally, MTX is the 4-amino, N-10 methyl analogue of folic acid. MTX is a cell specific antifolate analogue, which differs from folic acid in two substitutions, amino group and a methyl group. It is weak acid, slightly soluble in water and alcohol. MTX can be taken orally or administered by injection (subcutaneous, intramuscular, intravenous or intrathecal) ${ }^{(16)}$.

Folic acid plays a central role in a variety of metabolic reactions involving the transfer of one carbon units and is essential for cell replication. MTX is structurally related to folic acid and acts as an antagonist of folic acid. Once inside the cell, MTX either binds to its target enzymes, including dihydrofolate reductase "DHFR" or is polyglutamated by cytosolic folly polyglutamate synthase "FPGS" to methotrexate polyglutamate "MTXPG". The antifolate action is complex and includes inhibition of the enzyme "DHFR". Inhibition of "DHFR" leads to toxic effects through partial depletion of the tetrahydrofolate cofactors that are required for the synthesis of purines and thymidylate, and through direct inhibition of the folate-dependent enzymes of purine and thymidylate metabolism by the polyglutamates of MTX and dihydropolyglutamates that accumulate with DHFR inhibition ${ }^{(17)}$.

MTX functions as an inhibitor of enzymes, such as DHFR, aminoimidazole carboxamide ribonucleotide (AICAR) transferase, and thymidine synthase (TS), in folate metabolism. Down-regulation of these enzymes has negative effects on purine and pyrimidine synthesis, which impairs neurogenesis, including cell proliferation, survival, and differentiation, in the hippocampus. Additionally, MTX induces oxidative stress and DNA damage, which impedes various types of hippocampal neurogenesis ${ }^{(16)}$.

Stem and progenitor cells produce newborn neurons in two neurogenic regions: the subventricular zone (SVZ) of the lateral ventricle and the subgranular zone (SGZ) of the dentate gyrus (DG) in the hippocampus, a process that occurs continuously over a human or animal's lifespan. Newborn neurons in the SGZ migrate into granule cell layer (GCL) and become mature neurons, which are integrated into the hippocampal dentate gyrus circuit and enable hippocampal memory functions. As MTX induces aberrations related to hippocampal neurogenesis, it has a negative effect on hippocampus-dependent memory (18).

\section{II-Alpha-lipoic acid:}

Antioxidants are substances those are easily oxidized by reactive oxygen species (ROS) in a biological system, decreasing rate at which the ROS react with cellular components like lipid membranes, DNA or proteins. Alpha-lipoic acid (ALA) is a potent anti-oxidant that has been widely used in food supplement preparations. It was isolated from bovine liver in 1950, later on its chemical structure was established and confirmed by synthesis. ALA is not considered to be a vitamin and is synthesized in both the human and animal body from essential fatty acids. Now, it has become a common ingredient in multivitamin formulas and anti-aging supplements ${ }^{(\mathbf{1 9})}$. ALA is usually present in the mitochondrial matrix in the cells of organisms where cells metabolisms and energy production take place and functions as a cofactor for many mitochondrial enzymes involved in energy metabolism. It is readily absorbed from the diet, transported, taken up by a variety of cells and tissues and reduced to dihydrolipoic acid (DHLA).

ALA has been referred to as the "universal antioxidant" because of its unique antioxidant properties. Both, LA and DHLA are amphipathic molecules and act as antioxidants both in hydrophilic and lipophilic environments of any cell or tissue type, 
as well as any subcellular compartment, in the body as cytosol, the plasma membrane, serum and lipoproteins, while other well-known antioxidants work only in water (such as vitamin C) or work only in fatty tissues (such as vitamin E), thus it can work throughout the body. In addition, it is one of the very few substances that can actually cross the blood/brain barrier and do not exhibit any serious side effects ${ }^{(4)}$. ALA is a natural occurring dithiol compound with the chemical name of (1, 2-dithiolane-3-pentanoic acid) also known as thioctic acid. ALA is an 8-carbon fatty acid with sulfur atoms at C6 and C8 (Figure 4). Because the C6 carbon is chiral thus resulting in two possible optical isomers: R-LA and S-LA (20). The R-isomer is synthesized endogenously and bound to proteins but in therapeutic purposes, the $\mathrm{R}$ form is usually administered as a racemic mixture of R- LA and S-LA.

\section{Enantiomeric Forms of Lipoic Acid}

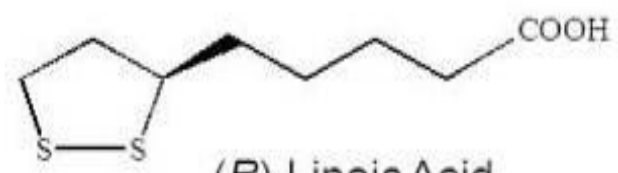

(R)-Lipoic Acid

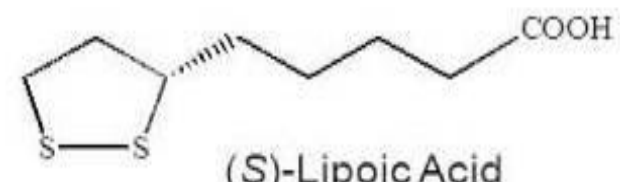

(S)-Lipoic Acid

Figure (4): The $\mathrm{R}$ and $\mathrm{S}$ enantiomers of lipoic acid ${ }^{(20)}$.

ALA is a natural compound synthesized enzymatically in the mitochondrion from octanoic acid and cysteine but only in small amounts that is bound to lysine amino acid forming lipoyl lysine, which is a food storage form of ALA. In addition to de novo synthesis, ALA can also be obtained from variety of food sources mostly kidney, heart, liver, spinach, broccoli, potatoes and some fruits as well as from nutritional supplements ${ }^{(\mathbf{2 1})}$. ALA can be adminstreted by intravenous (IV) or oral (tablets or capsules). ALA is most often prescribed orally for its antioxidant properties. The typical therapeutic oral dosages of ALA range from 50-600 mg daily. IV doses are most commonly used in the 300-600 $\mathrm{mg}$ range to reduce the risk of adverse effects ${ }^{(22)}$. Orally ingested ALA (tablets or soft gel capsules) is rapidly taken up in the gut in a $\mathrm{pH}$-dependent manner by monocarboxylic acid transporters (MCTs) and by the sodium-dependent multivitamin transporter (SMVT). The half-life of ALA in plasma is 30 min with bioavailability $~ 30 \%$ due to high liver extraction. It has been suggested that food intake reduces the bioavailability of ALA.
Therefore, it is recommended that ALA be taken 30 min before or $2 \mathrm{~h}$ after eating. ALA is bound to proteins in the body. Free lipoic acid is not present in the body unless administered therapeutically. It is currently believed that only the free, unbound form of ALA is responsible for the many therapeutic properties attributed to this antioxidant ${ }^{(4)}$. After rapid intestinal uptake it is readily taken up by a variety of cells and tissues (brain, heart, and muscle) with transient liver storage. In cells, ALA is rapidly reduced by various enzymes to dihydrolipoic acid (DHLA), which is rapidly excreted from cells. It is ALA and its metabolites that are rapidly excreted by filtration, primarily in the urine so ALA is not stored for longterm ${ }^{(22)}$. Neither animal nor human studies to date have any shown serious side effects with the ingestion of alpha-lipoic acid. Only at higher doses, possible side effects as nausea, diarrhea and flatulence are noticed ${ }^{(21)}$.

Alpha-lipoic acid is a powerful antioxidant that functions as an essential cofactor for mitochondrial pyruvate dehydrogenase and $\alpha$-ketoglutarate dehydrogenase leading to production of cellular energy. Because of its small size, its antioxidant activity extends to both its oxidized and reduced forms. Also, it can deliver antioxidant activity in both fat- and water-soluble mediums. Thus, effectively ALA can deliver its antioxidant effect to any cell or tissue type, as well as to any subcellular compartment, in the body ${ }^{(4)}$. The therapeutic actions of ALA is based on its antioxidant properties including its capacity in regenerating endogenous antioxidants, its metal chelating abilities, its action as a scavenger of reactive oxygen species (ROS) and its capability to repair oxidative damage ${ }^{(21)}$.

ALA, an essential cofactor for mitochondrial enzymes, is a natural antioxidant that readily crosses the blood-brain-barrier (BBB). It has been thought to be a potential candidate for the treatment of neuro degenerative disorders like Alzheimer's disease (AD) and Parkinson's disease (PD). ALA $(0.1 \%$ in diet) reduced the hippocampus-dependent memory deficit. ALA provides a wide range of activities, which can be helpful in neuro degenerative disorders. However, its effect on the cholinergic muscarinic receptors (M1M5) and its contribution in the emotional behaviour in Al-induced mouse model of neurotoxicity remains to be elucidated ${ }^{(23)}$.

\section{Administration:}

ALA plays a unique antioxidant role in the CNS because it has the ability to cross the blood-brain barrier, and it is taken up equally by the CNS and the peripheral nervous system due to both hydrophilic and lipophilic properties; thus, it can be absorbed and distributed easily into all body tissues ${ }^{(24)}$. Furthermore, ALA is a powerful antioxidant and neuroprotective agent that naturally occurs in the body and is also present in some foods ${ }^{(25)}$. There were also some 
studies on the effect of ALA on peripheral nervous system disorders. Melli et al. ${ }^{(26)}$ reported that ALA had a protective effect on mitochondrial damage and on neurotoxicity caused by chemotherapeutic agents. Ferreira et al. ${ }^{(27)}$ and Zhao et al. ${ }^{(28)}$ found that administration of ALA ameliorated cholinergic deficiency and improved spatial learning and memory performance in the MWM. The authors speculated that these beneficial effects may have been related to changes in monoamine (DA and NE) concentrations in the hippocampus. Additionally, Fava et al. (29) demonstrated that ALA may improve learning and memory in the animal hippocampus and cerebral cortex in numerous ways: (i) by affecting the neurotransmitter acetylcholine (Ach), (ii) by increasing glucose uptake (increasing ATP production), (iii) by inhibiting the formation of hydroxyl radicals, (iv) by scavenging ROS and thus downregulating inflammatory processes, (v) by scavenging lipid peroxidation products, and (vi) by inducing GSH synthesis enzymes.

Additionally, Zhao et al. (28) and Baluchnejadmojarad et al. ${ }^{(30)}$ concluded that ALA reverses cognitive dysfunction at least partly through its antioxidant properties. Additionally, Dixit et al. ${ }^{(31)}$ reported that ALA has a modulatory effect on apoptosis-associated proteins, as indicated by upregulation of $\mathrm{Bcl}-2$ and downregulation of $\mathrm{Bax}$, suggesting a positive influence of ALA on cell survival and the capability of ALA to stimulate various neuroprotective and repair pathways. Toklu $\boldsymbol{e t}$ al. ${ }^{(24)}$ and Emmez et al. ${ }^{(32)}$ demonstrated that ALA significantly increases tissue SOD and GSH activity. In addition, ALA increases de novo synthesis of cellular GSH by improving cysteine utilization. Moreover, ALA is recognized to restore antioxidants such as GSH, vitamin E and vitamin C. Newly reduced GSH and vitamin $\mathrm{C}$ also regenerate oxidized vitamin $\mathrm{E}$, forming an antioxidant network that protects the body from oxidative damage ${ }^{(33)}$. In addition, ALA is reduced to DHLA that is not destroyed by free radicals and can be recycled back into ALA. These two molecules are amphipathic and may act as antioxidants in both hydrophilic and lipophilic environments ${ }^{(24)}$. Also, ALA intake may reduce inflammatory markers through scavenging free radicals, down-regulating proinflammatory redox-sensitive signal transduction processes including nuclear factor kappa B translocation, leading to lowering release of other free radicals and cytotoxic cytokines ${ }^{(24)}$.

\section{CONCLUSION}

So, from this review article, it can be concluded that, alpha lipoic acid administration along with methotrxate therapy can ameliorate the harmfull effects of methotrxate on hippocampus.

Financial support and sponsorship: Nil.

Conflict of interest: Nil.

\section{REFERENCES}

1. Aggleton J (2012): Multiple anatomical systems embedded within the primate medial temporal lobe: Implications for hippocampal function. Neuroscience \& Biobehavioral Reviews, 36 (7): 1579-1596.

2. Rajitha $P$, Biswas $R$, Sabitha $M$ et al. (2017): Methotrexate in the treatment of psoriasis and rheumatoid arthritis: Mechanistic insights, current issues and novel delivery approaches. Current Pharmaceutical Design, 23 (24): 3550-3566.

3. Maritim A, Sanders R, Watkins J (2003): Effects of $\alpha$-lipoic acid on biomarkers of oxidative stress in streptozotocin-induced diabetic rats. The Journal of Nutritional Biochemistry, 14 (5): 288-294.

4. Rochette L, Ghibu S, Richard C et al. (2013): Direct and indirect antioxidant properties of $\alpha$-lipoic acid and therapeutic potential. Molecular Nutrition \& Food Research, 57 (1): 114-125.

5. Pearce J (2001): The effects of telencephalic pallial lesions on spatial, temporal, and emotional learning in goldfish. Journal of Neural Neurosurgery Psychiatry, 71 (3): 351-55.

6. Yau S, Gil-Mohapel J, Christie R et al. (2014): Physical exercise-induced adult neurogenesis: a good strategy to prevent cognitive decline in neurodegenerative diseases. BioMed Research International, 14: 1-21.

7. Silina E, Manturova N, Vasin V et al. (2020): Efficacy of A Novel Smart Polymeric Nanodrug in the Treatment of Experimental Wounds in Rats. Polymers (Basel), 12 (5): 1126-32.

8. Boccara C, Kjonigsen L, Hammer I et al. (2015): A three-plane architectonic atlas of the rat hippocampal region. Hippocampus, 25 (7): 838-857.

9. Amaral D, Witter M (1989): The three-dimensional organization of the hippocampal formation: a review of anatomical data. Neuroscience, 31 (3): 571-591.

10. Standring S (2008): Gray's Anatomy. Fortieth Ed. Churchill Livingstone Elsevier, the recent one, Pp: 349 - 351. https://www.scirp.org/ (S(351jmbntvnsjt1aadkposzje))/ reference/ ReferencesPapers.aspx?ReferenceID $=1830308$

11. Parekh R (2011): Interactions between oriens, basket and pyramidal cells during in vitro seizure-like events in CA1 of the rat hippocampus. Dissertation of Philosophy Doctor, Pennsylvania State University, Pp: 1-16. https://www.proquest.com/ openview/ f383096cf85c3727d2b49b7f027a5641/1?pqorigsite $=$ gscholar $\& \mathrm{cbl}=18750$

12. Andersen P, Morris R, Amaral D et al. (2007): The Hippocampus Book. Oxford University Press. The Hippocampal Formation, 7: 3-6.

13. Wyskiel D, Andrade $R$ (2016): Serotonin excites hippocampal CA1 GABAergic interneurons at the stratum radiatum-stratum lacunosum moleculare border. Hippocampus, 26: 1107-1114.

14. Duff M, Brown-Schmidt S (2012): The hippocampus and the flexible use and processing of language. Front Hum Neurosci., 6: 1-11.

15. Jacobs N, Allen T, Nguyen $\mathbf{N}$ et al. (2013): Critical role of the hippocampus in memory for elapsed time. $\mathbf{J}$ Neurosci., 33 (34): 13888-93.

16. Braga D, Chelazzi L, Grepioni F et al. (2016): Folic acid in the solid state: a synergistic computational, 
spectroscopic, and structural approach. Crystal Growth \& Design, 16 (4): 2218-2224.

17. Nogueira E, Sárria $M$, Azoia $N$ et al. (2018): Internalization of methotrexate conjugates by folate receptor- $\alpha$. Biochemistry, 57 (49): 6780-6786.

18. Kitabatake $Y$, Sailor $K$, Ming G et al. (2007) Adult neurogenesis and hippocampal memory function: new cells, more plasticity, new memories? Neurosurgery Clinics of North America, 18: 105-113.

19. Bilska A, Wlodek L (2005): Lipoic acid -the drug of the future? Pharmacol Rep., 57: 570-577.

20. Shay K, Moreau R, Smith E et al. (2009): Alphalipoic acid as a dietary supplement: molecular mechanisms and therapeutic potential. Biochimica et Biophysica Acta (BBA)-General Subjects, 1790 (10): 1149-1160.

21. Gorąca A, Kolega H, Piechota A et al. (2011): Lipoic acid- biological activity and therapeutic potential. Pharmacological Reports, 63:849-858.

22. Mignini F, Capacchietti M, Napolioni V et al. (2011): Single dose bioavailability and pharmacokinetic study ofa innovative formulation of $\alpha$ - lipoic acid (ALA600) in healthy volunteers. Minerva Med., 102 (6): 475-482.

23. Jamil A, Mahboob A, Ahmed T (2016): Ibuprofen targets neuronal pentraxins expresion and improves cognitive function in mouse model of $\mathrm{AlCl} 3$-induced neurotoxicity. Experimental and Therapeutic Medicine, 11 (2): 601-606.

24. Toklu H, Hakan T, Celik H et al. (2010): Neuroprotective effects of alpha-lipoic acid in experimental spinal cord injury in rats. The Journal of Spinal Cord Medicine, 33 (4): 401-409.

25. Tomassoni D, Amenta F, Di Cesare L et al. (2013): Neuroprotective activity of thioctic acid in central nervous system lesions consequentto peripheral nerve injury. Bio Med Research International, 13:985093.

26. Melli G, Taiana M, Camozzi F et al. (2008): Alphalipoic acid prevents mitochondrial damage and neurotoxicity in experimental chemotherapy neuropathy. Experimental Neurology, 214 (2): 276-284.

27. Ferreira P, Militão G, Freitas R (2009): Lipoic acid effects on lipid peroxidation level, superoxide dismutase activity and monoamines concentration in rat hippocampus. Neuroscience Letters, 464 (2): 131-134

28. Zhao R, Xu F, Xu X et al. (2015): Effects of alphalipoic acid on spatial learning and memory, oxidative stress, and central cholinergic system in a rat model of vascular dementia. Neuroscience Letters, 587: 113-119.

29. Fava A, Pirritano D, Plastino M et al. (2013): The effect of lipoic acid therapy on cognitive functioning in patients with Alzheimer's disease. Journal of Neurodegenerative Diseases, 13: 454253.

30. Baluchnejadmojarad T, Roghani M, Kamran M et al. (2012): The Effect of Alpha-Lipoic Acid on Learning and Memory Deficit in a Rat Model of Temporal Lobe Epilepsy. Basic and Clinical Neuroscience, 3 (3): 58-66.

31. Dixit S, Dhar P, Mehra R (2015): Alpha lipoic acid (ALA) modulates expression of apoptosis associated proteins in hippocampus of rats exposed during postnatal period to sodium arsenite (NaAsO2): Toxicology Reports, 2: 78-87.

32. Emmez H, Yildirim Z, Kale A et al. (2010): Antiapoptotic and neuroprotective effects of alpha-lipoic acid on spinal cord ischemia-reperfusion injury in rabbits. Acta Neurochirurgica, 152 (9): 1591-1601.

33. Abdel-Wahab B, Metwally M (2014): Protective effect of alpha lipoic acid against lead-induced hippocampal neurotoxicity and neuronal oxidative stress in rats. Austin J Pharmacol Ther., 2 (11): 8-12.

34. Wong B, Brown D, Pan T et al. (2001): Oxidative impairment in scrapie-infected mice is associated with brain metals perturbations and altered antioxidant activities. Journal of Neurochemistry, 79 (3): 689-698. 